\title{
ATENDIMENTO A PESSOA IDOSA NOS SERVIÇOS DE SAÚDE \\ - percepção dos profissionais de saúde
}

\author{
Edméia Campos Meira ${ }^{a}$ \\ Maria do Rosário de Menezes ${ }^{b}$ \\ Andréa dos Santos Souza ${ }^{c}$ \\ Maria Aparecida Cabral Tavares de Santana ${ }^{d}$ \\ Jackeline Aparecida Leite Oliveira ${ }^{\mathrm{e}}$ \\ Alice Almeida Lima ${ }^{\mathrm{e}}$ \\ Larissa Campos Meira ${ }^{f}$ \\ Luciana Araújo dos Reis ${ }^{g}$
}

\begin{abstract}
Resumo
(Objetivo) avaliar a opinião dos profissionais de saúde sobre o atendimento prestado a pessoa idosa nos serviços de saúde da Atenção Básica. (Metodologia) trata-se de estudo descritivo, exploratório, de natureza qualitativa, realizado nos serviços de saúde do Município de Jequié-BA, sendo a amostra constituída de trinta profissionais de saúde. Os dados foram tratados por meio da análise de conteúdo de Bardin. (Resultados) a maioria dos entrevistados era do sexo feminino, encontra-se no grupo etário de 31 a 40 anos e eram graduados em Enfermagem. A análise dos resultados sobre o atendimento possibilitou a identificação de três categorias temáticas: 1- Motivação para o Comparecimento ao Serviço de Saúde; 2- Dificuldades de Acesso; 3- Acolhimento como Estratégia do Processo de Trabalho. (Conclusões) este estudo permitiu uma análise das questões que permeiam o atendimento a pessoa idosa, assim, o qual irá contribuir na promoção de reflexão da situação de saúde pelos profissionais que atuam nas práticas do cuidado, além de incentivar medidas inovadoras e criativas nos enfrentamentos de desafios que violam o direito da pessoa idosa.
\end{abstract}

Palavras-chave: Profissionais de saúde; Assistência a saúde; Cidadania do idoso; Violência institucional.

a. Enfermeira. Mestre em Enfermagem. Docente Adjunta do Departamento de Saúde da Universidade Estadual do Sudoeste da Bahia/UESB. Graduada em Odontologia pela Escola Bahiana de Medicina e Saúde Publica

b. Enfermeira. Doutora em Interunidades Saúde do Adulto Idoso. Docente Adjunta do Departamento de Enfermagem da Universidade Federal da Bahia/UFBA.

c. Enfermeira. Mestre e doutoranda em Enfermagem/UFBA. Docente Adjunta do Departamento de Saúde da UESB.

d. Assistente Social. Doutora em Saúde Pública. Assistente Social do Hospital Prado Valadares.

e. Enfermeira/UESB.

f. Enfermeira/Faculdade de Ciências e Tecnologia.

g. Fisioterapeuta. Doutora em Ciências da Saúde. Docente Adjunta do Departamento de Saúde da Universidade Estadual do Sudoeste da Bahia/UESB. 


\title{
THE ELDER CARE SERVICES IN HEALTH \\ - perception of health
}

\begin{abstract}
(Objective) to assess the views of health professionals on the care provided to elderly people in the health services from Primary Care. (Methodology) this is a descriptive, exploratory study, qualitative in nature, carried in the health services of the Municipality of Jequié-BA, the sample consists of thirty health professionals. The data were processed through the content analysis of Bardin. (Results) Most respondents were female, is in the age group 31 to 40 years and were graduates in Nursing. The results on the care enabled the identification of three themes: 1 - Motivation for Attendance Health Service; 2 - Difficulties of access; 3 - Home and Work Process Strategy. (Conclusions) This study provided an analysis of the issues that permeate the care of the elderly, as well, which will help in promoting reflection of the situation of health professionals who work in care practices, and encourage innovative and creative measures in confrontations challenges that violate the right of the elderly.
\end{abstract}

Keywords: Health professionals; Health assistance; Citizenship of the elderly; Institutional violence.

\section{LOS SERVICIOS DE CUIDADO DE ANCIANOS EN LA SALUD \\ - la percepción de la salud.}

\begin{abstract}
Resumen
(Meta) evaluar las opiniones de los profesionales de la salud en la atención prestada a las personas mayores en los servicios de salud en la atención primaria. (Metodología) es un estudio cualitativo descriptivo, exploratorio llevado a cabo en los servicios de salud en el municipio de Jequie-BA, la muestra estuvo conformada por treinta profesionales de la salud. Los datos fueron tratados por medio del análisis de contenido de Bardin. (Resultados) la mayoría de los encuestados eran mujeres, es en el grupo de edad de 31-40 años y se habían graduado en Enfermería. Los resultados en el cuidado permitieron identificar tres temas: 1 - Motivación por la asistencia del Servicio de Salud; 2 - Dificultades de acceso; 3 - Shelter como la estrategia del proceso de trabajo. (Conclusiones) este estudio permitieron un análisis de los aspectos que impregnan el cuidado de personas de edad avanzada, así, lo que ayudará en la promoción de la reflexión de la situación de los profesionales de la salud que trabajan en las prácticas de atención y estimular acciones innovadoras y creativas en enfrentamientos retos a los que violan el derecho de las personas de edad avanzada.

Palabras clave: Profesionales de la salud; El cuidado de la salud; La ciudadanía de las personas mayores; Violencia institucional.
\end{abstract}




\section{INTRODUÇÃO}

O crescimento da população de idosos é uma realidade emergente e está ocorrendo de forma bastante acelerada, não só no Brasil, mas em todo o mundo, desta forma, há a necessidade de um olhar diferenciado a estas pessoas que a cada dia envelhece e cuja expectativa de vida é cada vez mais longa. No Brasil, a Pesquisa Nacional por Amostra de Domicílios revelou que a quantidade de pessoas com mais de 60 anos somava cerca de 21 milhões. ${ }^{(1,2)}$

Atrelado à transição demográfica percebe-se que com o passar dos anos ocorreram modificações no perfil epidemiológico do Brasil configurando um aumento das doenças crônicas, comuns nas idades avançadas. Esse fenômeno traz repercussões profundas no contexto saúde-doença, acarretando como um dos resultados a utilização crescente das pessoas idosas por serviços de saúde. ${ }^{(3)}$

Ao buscar o serviço de saúde, a pessoa idosa poderá confrontar com situações que dificultam o seu atendimento. Ao analisar o setor saúde são visíveis as modificações pelas quais passam a prestação da assistência, verificando a falta de equidade, eficiência e qualidade. ${ }^{(4)}$

A complexidade do cuidado necessário para o atendimento requer reordenação dos serviços de saúde, o que reforça a necessidade de investimento no aperfeiçoamento de recursos humanos. Nesse sentido, faz-se necessário equipes multiprofissionais, coesas no agir interdisciplinarmente com enfoque na ciência geronto-geriátrica, respeitando as diferenças individuais e potencialidades do ser que envelhece e do processo de envelhecimento humano, para intervir não somente no processo patológico, mas também, no reconhecimento do idoso como um cidadão, e como tal provido de direitos e deveres. ${ }^{(5)}$

A discussão dos direitos da pessoa idosa, no Brasil já é uma realidade que está presente há vários anos e vem acentuando-se devido ao reconhecimento da transição demográfica. Dessa maneira, Políticas Públicas de Atenção ao Idoso são instrumentos que garantem a proteção a esse gru- po populacional, reconhecendo-os como cidadãos protagonistas que, devem ser considerados parceiros ativos necessários ao desenvolvimento dessas políticas.

Entretanto, a operacionalização de uma política bem como das demais ações empreendidas no campo assistencial ocorrem de forma descentralizada, assim como a articulação com as demais políticas voltadas para a pessoa idosa. ${ }^{(6)}$ As dificuldades na implementação das políticas para o idoso devem-se à precária captação de recursos, ao delicado sistema de informação para análise de condições de vida e saúde atreladas à falta de capacitação dos profissionais. ${ }^{(7,8)}$

Diante das evidentes necessidades de saúde apresentadas pelos idosos e da importância do compromisso dos profissionais de saúde frente a tais necessidades, este estudo tem como objetivo avaliar a opinião dos profissionais de saúde sobre o atendimento prestado a pessoa idosa nos serviços de saúde da Atenção Básica em Jequié-BA.

\section{METODOLOGIA}

Trata-se de pesquisa multicêntrica, de caráter descritiva e exploratória, com abordagem qualitativa, originária do Projeto de Pesquisa da Universidade Estadual do Sudoeste da Bahia em parceria com a Universidade Federal da Bahia: "O Difícil Encontro Entre a Não Violação dos Direitos e Assistência de Idosos nos Serviços de Saúde: percepção dos profissionais de saúde sobre o atendimento e assistência a pessoas idosas".

O cenário de estudo foram 4 Unidades de Saúde da Família e 2 Centros de Saúde, no Município de Jequié-BA. O município disponibiliza diversos serviços de saúde à sua população e às cidades circunvizinhas, possuindo na rede básica municipal de saúde 44 unidades de saúde, 4 Centros de Saúde e 18 Unidades de Saúde da Família e 21 Postos de Saúde. ${ }^{(9)}$ 
A amostra do estudo foi constituída de trinta profissionais da estratégia de Saúde da Família entre eles: 06 enfermeiros, 06 técnicos de enfermagem, 02 médicos, na faixa etária entre 20 a 60 anos de idade, sendo a maioria do sexo feminino.

Para a coleta de dados foi utilizado uma entrevista composta de duas partes: a primeira contém questões objetivas relacionadas à identificação dos informantes. A segunda consta de questões abertas que possibilitam a emissão de opiniões dos profissionais de saúde relacionadas à especificidade do atendimento aos idosos nos serviços de saúde, sendo o período de coleta de dados janeiro a março de 2013.
Os dados foram analisados através da análise de conteúdo de Bardin ${ }^{(10)}$ e a pesquisa foi aprovada Comitê de Ética em Pesquisa (CEP) da UESB sob o protocolo $\mathrm{n}^{\circ} 203$ de 2009 .

\section{RESULTADOS E DISCUSSÃO}

A partir das informações fornecidas pelos sujeitos do estudo sobre o atendimento prestado ao idoso nos serviços de saúde, emergiram no total três categorias temáticas com suas respectivas subcategorias.

Quadro 1 - Apresentação das categorias e subcategorias temáticas, Jequié/BA, 2013

\begin{tabular}{l|l}
\hline \multicolumn{1}{c|}{ CATEgorias } & \multicolumn{1}{c}{ SubCATEGORIAS } \\
\hline 1. Motivação para o Comparecimento ao Serviço de & Necessidades de Controle da Doença \\
\cline { 2 - 2 } Saúde - MCSS & Necessidades Psico/sociais \\
\hline \multirow{3}{*}{ 2. Dificuldades de Acesso ao Serviço de Saúde - DASS } & Condições Geográficas do Território \\
\cline { 2 - 2 } & Fragilização do Envelhecimento Humano \\
\cline { 2 - 2 } & Problemas no Acesso Socio-Organizacional \\
\hline \multirow{2}{*}{$\begin{array}{l}\text { 3. Acolhimento como Estratégia do Processo de } \\
\text { Trabalho - AEPT }\end{array}$} & Avanços na Consolidação do Acolhimento \\
\cline { 2 - 2 } & Limitações na Consolidação do Acolhimento \\
\hline
\end{tabular}

\section{CATEGORIA 1 - Motivação para o}

Comparecimento ao Serviço de Saúde

Desse modo, baseado nos relatos dos sujeitos deste estudo, identificou-se alguns motivos pelos quais os idosos procuram o serviço de saúde a partir dos quais emergiram duas subcategorias: $\mathrm{Ne}$ cessidades de Controle da Doença e Necessidades Psico/sociais.

Na sub-categoria "Necessidades de Controle da Doença", pode-se verificar que o processo de enveIhecimento é marcado por fatores socioeconômicos, ambientais e pelo aparecimento de enfermidades, sendo influenciado não apenas pela idade, mas, em grande medida, pelo modo como o indiví- duo vive. Sabemos que a probabilidade de adoecer aumenta com a idade, devido a mudanças biológicas tornando o organismo mais vulnerável a várias doenças. ${ }^{(11,12)}$ Percebe-se que alguns dos entrevistados referiram que o motivo que leva a pessoa idosa a procurar o serviço de saúde é a necessidade de controle da doença. Podemos constatar a veracidade dessa afirmação com as unidades de análise dispostas:

São os problemas referentes à própria idade, como hipertensão, diabetes... (Profissional 1) 
A maioria deles é hipertenso, diabético, vem pra acompanhar a doença... (Profissional 14)

Só pra procurar o serviço de hipertensão atrás de remédio né, remédio pra dor de coluna, de doença... (Profissional 10)

... Outros vêm pegar só a medicação ai depois a gente orienta que tem um serviço [...]. (Profissional 4)

A necessidade de controle da doença foi relatada pela maioria dos profissionais, tal como controle das doenças crônicas, a exemplo da hipertensão arterial e diabetes, em que a maioria dos idosos apresenta tais doenças, exigindo acompanhamento constante, e consequentemente bastante gastos/custos, medicação contínua e exames periódicos, o que aumenta a utilização dos serviços de saúde.(6) Os trabalhos voltados à prevenção de tais doenças possibilitam melhoria da qualidade de vida dos idosos, além de causar uma diminuição em recursos financeiros e humanos, uma vez que o cuidado voltado a pessoas portadoras de tais doenças configura-se como sendo de alto custo tanto para as famílias como para o Estado. ${ }^{(6)}$

Diante das unidades de análise, fica explícito nas falas destes profissionais à procura do serviço com fins exclusivamente de acompanhamento e tratamento de doenças. O modelo assistencial que opera hoje nos serviços é centralmente organizado a partir dos problemas específicos, dentro da ótica hegemônica do modelo médico biologicista, subordinando à dimensão cuidadora do profissional a um papel irrelevante e complementar. ${ }^{(13)}$

Na sub-categoria "Necessidades Psico/sociais", os profissionais de saúde disseram que os motivos que levam a pessoa idosa a procurar o serviço de saúde são as necessidades que consideram o trabalho de educação em saúde na comunidade, na sala de espera, necessidade de uma comunicação participativa da satisfação com o atendimento realizado. Conforme descrito a seguir:

... Devido ao trabalho de educação em saúde na comunidade fui passando a importância deles frequentar a unidade de saúde, fazer o acompanhamento... [...] eles têm muita necessidade de falar sobre as suas doenças e sobre a sua família. (Profissional 2)

... Porque não tem nada a fazer, tempo ocioso, fica conversando, vem pra distrair... aqui não tem grupo, tem um projeto da UESB que uma boa parte da comunidade faz parte, eles tão amando... (Profissional 6)

... Porque eles gostam de bater papo [...] a gente percebe que existe uma satisfação, desabafar, contar os problemas pessoais... (Profissional 12)

Nos relatos dos profissionais envolvidos percebe-se que a relação estabelecida entre o profissional-idoso, é um elemento de fundamental importância no espaço do atendimento, podendo influenciar o seu modo de agir e pensar, no qual permeiam espaços de diálogos, co-responsabilização, relações de acolhimento e confiança. Critérios essenciais para o desenvolvimento da motivação entre ir ou não ao atendimento de saúde.

A pessoa idosa ao procurar um serviço de saúde, não está apenas buscando o exame, mas também de outras necessidades interpessoal, tais como: afeto, atenção, o interlacionamento como estratégia do atendimento integral e equânime, caracterizando o uso de tecnologias leves. ${ }^{(13)}$

CATEGORIA 2 - Dificuldades de Acesso ao Serviço de Saúde

Esta categoria apresenta à compreensão dos profissionais de saúde quanto às dificuldades de acesso a unidade de saúde. Entende-se por acesso à distância da unidade de saúde e o local de moradia do indivíduo, tempo e meios utilizados para o deslocamento, as dificuldades enfrentadas para a obtenção do atendimento, tratamento recebido pelo usuário, priorização de situações de risco, respostas obtidas para demandas individuais e coletivas. ${ }^{(14)}$ Segundo Bahia ${ }^{(15)}$ o acesso tem dois componentes: o acesso geográfico e o acesso sócio-organizacional. 
Os profissionais de saúde ao serem questionados sobre as dificuldades de acesso da pessoa idosa ao serviço de saúde relataram que a maioria dos idosos encontra algum tipo de dificuldade. Identificamos nas falas dos entrevistados as seguintes subcategorias: Condições Geográficas do Território e Fragilização do Envelhecimento Humano.

Quanto a sub-categoria "Condições Geográficas do Território" são caracterizadas nas respostas dos entrevistados, principalmente, como entrave para a acessibilidade utilizadas pelas pessoas idosas estão as dificuldades geográfica física e climática, pode-se observar nas unidades de análise que seguem:

... O terreno é acidentado, tem ladeira, tem pedra, é buraco, não é calçado... (Profissional 3)

... Tem a questão das ruas que não são adequadas, a dificuldades deles são essas, é transpor essas barreiras arquitetônicas pra chegar à unidade [...], seria melhor se a Unidade fosse mais centralizada... (Profissional 8)

[...] Eles dizem que o sol tá quente... (Profissional 11)

... A unidade fica muito descentralizada, privilegiando mais o pessoal da equipe II, aí eles falam que ficam um pouco longe. (Profissional13)

Tal dificuldade decorrente da geografia física é relacionada ao relevo da área onde se situa o $\mathrm{Mu}$ nicípio de Jequié por ser bastante acidentado devido à presença de planícies, morros e serras. ${ }^{(9)}$ Aliado à geografia do município, nota-se, também em algumas falas as dificuldades relacionadas com a infraestrutura inadequada de algumas ruas por não serem calçadas, configurando em grandes dificuldades para o idoso. Como se não bastassem, algumas Unidades de Saúde também não são devidamente centralizadas, ficando distante do local de sua moradia. Também foi citado como dificuldade o fator climático que se deve ao fato de o município de Jequié situar-se na região sudeste do Estado da Bahia, especificamente entre a Zona da Mata e
Caatinga, fato este que the confere um clima quente. ${ }^{(9)}$

A pessoa idosa ao procurar estes serviços enfrenta diversas barreiras, a primeira está em como chegar ao serviço, pois se sabe que para muitos o meio de transporte é bastante difícil. Critérios como proximidade, acessibilidade, custo-efetividade e boa adaptação do usuário ao serviço de saúde são instrumentos que viabilizarão o bem estar para os idosos e toda a comunidade.(1) Cabe aos gestores do SUS estabelecer em suas áreas de abrangência de serviços a articulação de parcerias para implantar ações que permitam e/ou viabilize o deslocamento do cidadão idoso, sobretudo àquele que já apresenta dificuldades de locomoção, de modo a auxiliar na manutenção e no apoio à independência funcional da pessoa idosa. ${ }^{(15)}$

Quanto à subcategoria "A Fragilização do EnveIhecimento Humano" foi apontada pelos sujeitos da pesquisa como dificuldades de acesso ao serviço de saúde. A fragilização pode está ligada a algumas características: ser maior de 80 anos, presença de pluripatologia, isolamento social, transtorno cognitivo, afetivo, dificuldade de deambulação, incontinência urinária. ${ }^{(16)} \mathrm{O}$ que se pode verificar nas seguintes afirmações:

Pois não tem mais aquela locomoção, tem problema de saúde ne, problema nas pernas... (Profissional 3)

... Tem alguns que não verm devido à dificuldade de deambular [...] vem com muleta, então alguns tem deficiência visual...(Profissional 5)

Tem um grande número de pessoas acamadas que nós não conseguimos estar atendendo todo mundo. (Profissional 7)

As doenças comumente associadas ao enveIhecimento compreendem as cardiovasculares, as respiratórias, as neurológicas, as metabólicas, as sensoriais e as músculos esqueléticas (artrose/osteoartrose/osteoartrite, dores lombares, dentre outras), como podemos verificar esta última foi bas- 
tante citada acima como fator que dificulta e/ou impossibilita a ida ao serviço. ${ }^{(15)}$

Pensando nestes agravos que, deste 1999, quando foi promulgada a Política Nacional do Idoso traz como propósito a promoção do envelhecimento saudável, voltado ao desenvolvimento de ações que orientam os idosos e as pessoas que estão envelhecendo quanto à importância da adoção de hábitos saudáveis de vida, a eliminação de comportamentos prejudicial à saúde, além de orientação aos idosos e seus familiares quanto aos riscos ambientais que favorecem as quedas. ${ }^{(11)}$

Quanto a sub-categoria: "O acesso sócio-organizacional" relaciona-se às características e recursos que facilitam ou impedem os esforços das pessoas em receber os cuidados de uma equipe de saúde. (14) Identificamos neste estudo os pontos que foram considerados fatores que dificultam o atendimento e estão interligados aos problemas funcionais do processo de trabalho, tais como, falta de recursos materiais, falta de recursos humanos e comunicação ineficaz. Identificados nas afirmações a seguir:

... O sistema ta fora do ar... (Profissional 14) ... Não estão tendo atendimento médico por falta de carro para visita (...) eu no caso como enfermeira eu graças a Deus faço minhas visitas, mas precisa ter a do médico e tem alguns meses que eles não recebem esta visita... (Profissional 10)

... Faltam materiais, os aparelhos demoram de ser consertados... (Profissional 4)

A dificuldade que eu observo é a marcação para clinico (...) como eu to te falando esta faltando recursos humanos (...) às vezes a prefeitura não paga ai o médico não vem, esse idoso vem, ai o médico não atende ai fica desacreditado, aborrecido... (Profissional 12)

... Então a gente tem deficiência de pessoal e de pessoal preparado para atender... (Profissional 9)

... É a queixa que eles falam, então na maioria das vezes eles não dá a informação precisa.... (Profissional 1)
Em alguns momentos da observação direta em campo, assistimos aos seguintes entraves: o usuário buscava o serviço de saúde para procedimentos, aquisição de medicamentos e outros materiais necessários, mas não o encontravam. Também foi observado a não realização de visitas/consultas domiciliares para os usuários dependentes/fragilizados impossibilitados de ir a unidade de saúde. As tecnologias duras como preconiza Mehy ${ }^{(17)}$ encontram-se sem manutenção para a devida operacionalização de procedimentos básicos de saúde, propiciando uma demanda reprimida. Fato este que chama a atenção, porque a maioria dos usuários era idoso que já tinham dificuldades de acessibilidade.

Nos relatos dos profissionais transparece a angústia e um sentimento de impotência em desenvolver o trabalho baseado no princípio da integralidade preconizado pelo SUS. Tais fatores interferem para a baixa resolutividade e precário atendimento, gerando um sentimento de "frustração" no desempenho do profissional de saúde e insatisfação por parte da pessoa idosa, tornando a relação trabalhador-usuário conflituosa e desgastante para o profissional.

Como há uma centralização dos recursos financeiros na Secretária Municipal de Saúde, os profissionais sentem os reflexos dessa escassez de recursos no âmbito operacional. Ainda observa-se em campo uma preocupação de alguns profissionais de saúde em reivindicar, em trazer a problemática para os espaços coletivos, porém poda logo tal pensamento com "medo" de represália e consequentemente perda do emprego, já que alguns são cargos comissionados, e mesmo quando concursados relataram que o concurso não proporciona a estabilidade que constitucionalmente é de direito.

De acordo com a Portaria 648/MS, compete às Secretarias Municipais de Saúde e ao Distrito Sanitário garantir infra-estrutura necessária ao funcionamento das Unidades Básicas de Saúde, dotando-as de recursos materiais, equipamentos e insumos suficientes para o conjunto de ações propostas. ${ }^{(17)}$ Entretanto, o financiamento fica restrito aos gestores (prefeitos e secretários de saúde), necessitando 
de uma participação efetiva dos profissionais de saúde e usuários nas decisões e deliberação realizadas por tal secretaria.

No presente estudo, observou-se nas falas dos profissionais a violência institucional, quando citaram a problemática. Assim, a violência institucional "relaciona-se àquela exercida pelos serviços públicos, por ação ou omissão, podendo incluir desde a falta de acesso à saúde até a má qualidade dos serviços. ${ }^{\left({ }^{16)}\right.}$ Abrange abusos cometidos em virtude das relações de poder desiguais entre usuários e profissionais dentro das instituições, atendimento, muitas vezes negligente, inadequada estrutura dos órgãos públicos, os quais não são apropriados, dificultando, dessa maneira o acesso da pessoa idosa. ${ }^{(18)}$

\section{CATEGORIA 3 - Acolhimento como}

Estratégia do Processo de Trabalho

Assim, baseados nos relatos, identificamos o AcoIhimento como Estratégia do Processo de TrabaIho, a partir das quais emergiram duas subcategorias: Avanços na Consolidação do Acolhimento e Limitações na Consolidação do Acolhimento.

$\mathrm{Na}$ sub-categoria: "Avanços na Consolidação do Acolhimento", os entrevistados referiram a noção do Acolhimento relacionado tanto com a resolutividade, responsabilização, relação humanizada, quanto a produção na construção de consensos entre os profissionais. Pode-se constatar esta afirmação com as unidades de análise apresentadas a seguir:

... Quando a gente ta aqui circulando pegando o cartão, a gente vai chegando logo e acomodando eles... (Profissional 7)

... Eu procuro sempre saber dos seus problemas, porque veio, o que esta sentindo... (Profissional 13)

... As queixas são ouvidas, atende com mais cautela, temos um cuidado especial, a gente vai explicando, vendo se entendeu... (Profissional 4)
Nós procuramos acolher da melhor forma possivel, inclusive nós discutimos isso nas reuniões que fazemos semanalmente, (...) para que todos estejam envolvidos em dá um bom acolhimento aos idosos... (Profissional 2)

A concepção aqui entendida de acolhimento que permeia os discursos dos trabalhadores é a de que este tem o objetivo de garantir respostas às necessidades dos usuários que não sejam necessariamente clínicas, mas que diz respeito ao acolhimento como uma atitude diante do usuário, de escuta e responsabilização proporcionando respostas às necessidades deste idoso. Os profissionais passam a recebê-los de outra forma, com mais espaço para o diálogo, e os idosos sente-se mais "acolhidos", o que diminui a ocorrência de conflitos e discussões, mesmo havendo ainda espera e falta de vagas. Sobre este modo de acolher há a abordagem do acolhimento enquanto um modo de se relacionar com os usuários e o define como uma "rede de conversações" propõe assim a adoção do termo acolhimento-diálogo para definir um tipo especial de conversa que se dá/ou deveria ocorrer no interior dos serviços de saúde. ${ }^{(19)}$

Observa-se o destaque para a humanização como uma mudança positiva e resultante da inserção do acolhimento na dinâmica do trabalho no serviço. As mudanças apontadas nas entrevistas parecem indicar que o acolhimento, do modo como se estruturou entre estes profissionais, contribuiu para "humanizar" as ações de recepção dos usuários e suas demandas no serviço. Percebe-se também nas falas dos profissionais que ocorrem reuniões entre a equipe de saúde, para avaliar a forma como ocorre ou deveria ocorrer o acolhimento.

Temos, então, duas possibilidades de entendimento do acolhimento expostas aqui: uma como postura diante do usuário e suas necessidades, de contínua investigação das necessidades de saúde e o tratamento em que está baseada a humanização, e outra, como dispositivo capaz de reorganizar o trabalho na unidade, ampliando os espaços democráticos de discussão, e decisões coletivas. 
Desenvolver a implantação do acolhimento com a participação dos trabalhadores da unidade e dos usuários, com múltiplos espaços de discussão e pactuação dessa inovação no processo de trabalho, mediante reuniões internas da equipe, reuniões com usuários nas salas de espera, informação à comunidade, é de grande importância para avaliar e adequar a implementação desta inovadora ferramenta. ${ }^{(20)}$

$\mathrm{Na}$ subcategoria: Limitações na Consolidação do Acolhimento, identificamos neste estudo, segundo relato de $43 \%$ dos trabalhadores entrevistados, os pontos que foram considerados como fatores limitantes na consolidação do acolhimento, tais como: acolhimento como dimensão espacial, acolhimento como uma ação de triagem e falta de humanização no cuidado, como podemos verificar nas seguintes afirmações:

... Às vezes a gente fica muito na sala, tô muito aqui ai quem faz o acolhimento é o pessoal da recepção, precisa melhorar no acolher... (Profissional 2)

... Eu acho que o pessoal é bem acolhido, as meninas que ficam na recepção eles atendam bem... (Profissional G)

Na realidade a gente faz a triagem, ou seja, o idoso chega ele procura uma de nós (técnicos de enfermagem) de cada equipe e diz o que esta sentindo, ai nós analisamos vemos a necessidade.... (Profissional 9)

... A maioria das pessoas não está preparada para atender não só o idoso, mas toda a clientela, mas o idoso em particular (...) então falta direcionar, questionar ao idoso... (Profissional 4)

Ainda na visão de alguns trabalhadores o acolhimento é entendido como tendo um local específico para realizá-lo, neste caso o local seria a recepção. Tradicionalmente, a noção de acolhimento no campo da saúde tem sido identificada, ora como uma dimensão espacial, que se traduz em recepção administrativa, ora como uma ação de triagem administrativa e repasse de encaminhamentos para serviços especializados. Ambas as noções têm sua importância, entretanto, quando tomadas isoladamente dos processos de trabalho em saúde, se restringem a uma ação isolada e descomprometida com os processos de responsabilização e produção de vínculo. ${ }^{(20)}$

Desta forma, o acolhimento não é um espaço ou um local, mas uma postura ética, não pressupõe hora ou profissional específico para fazê-lo, implica compartilhamento de saberes e invenções, tomando para si a responsabilidade de resolver os problemas do usuário, com responsabilidade e resolutividade. ${ }^{(20)}$

Ainda avaliando as limitações na consolidação do acolhimento, outro enfoque identificado ao acoIhimento foi o da realização de triagem, a função de determinado profissional era basicamente escutar a queixa e avaliar a necessidade do usuário e decidir que caminho ele vai seguir. Um profissional de saúde, na maioria das vezes, foi designado como o trabalhador responsável pelo acolhimento, enquanto etapa do processo de trabalho, neste caso, era sempre os técnicos de enfermagem. No entanto, é preciso estar atento para a diferença entre triagem e acolhimento, pois este último não se constitui como uma etapa do processo, mas como ação que deve ocorrer em todos os locais e momentos do serviço de saúde. ${ }^{(20)}$

\section{CONCLUSÃO}

Diante das falas dos profissionais entrevistados verificamos que os mesmo avaliam a assistência prestada ao idoso na atenção básica como adequada, embora segundo os mesmos algumas dificuldades ainda precisam ser superadas no intuito de promover uma melhor assistência. Dentre estas dificuldades destaca-se a necessidade da construção de espaços que promovam modos de viver mais salutares em todas as etapas de vida, favorecendo a prática de atividade física e ações educativas em saúde. 


\section{REFERÊNCIAS}

1. Kalache A, Hoskins I, Mende S. Hacia uma atención primaria de salud adaptada a las personas de edad. Ver. Panam. salud pública 2005; 17(5-6): 444-451.

2. IBGE. Pesquisa nacional por amostra de domicílios. Rio de Janeiro: 2008. Disponível em: http://www.ibge.gov.br/home/estatistica/ populacao/trabalhoerendimento/pnad2008/

3. default.shtm.

4. Veras R. Envelhecimento populacional contemporâneo: demandas, desafios e inovações. Rev. saúde pública, 2009;43(3):548554.

5. Organização Pan-Americana da Saúde. Desempenho em equipes de saúde. Rio de Janeiro: Editora FGV; 2001. Disponível em: http://www.opas.org.br/rh/publicacoes/textos/ pdrh_des.pdf

6. Sayeg MA, Mesquita RAV, Costa NE. Políticas públicas de Saúde para o Envelhecimento. In: Freitas EV et al. Tratado de geriatria e gerontologia. Rio de Janeiro: Guanabara Koogan; 2006. p. 1394-1401.

7. Camarano AA, Pasinato MT. O envelhecimento populacional na agenda das políticas públicas. In: Camarano, A.A. Os novos idosos brasileiros: muito além dos 60? Organizado por Ana Amelia C. Rio de Janeiro: IPEA; 2004. p.1-4O.

8. Motta L B, Aguiar AC. Novas competências profissionais em saúde e o envelhecimento populacional brasileiro: integralidade, interdisciplinaridade e intersetorialidade. Ciência e saúde. 2007;12(2):363-372.

9. Martins JJ et al. Políticas públicas de atenção à saúde do idoso: reflexão acerca da capacitação dos profissionais da saúde para o cuidado com o idoso. Rev. bras. geriatr. gerontol. 2007;10(3):1-12.

10. Jequié. Secretaria Municipal de Saúde. Plano Municipal de Saúde de Jequié para o exercício de 2006-2009. Jequié; 2006.

11. Bardin L. Análise de conteúdo. Lisboa: Edições 70; 2007.

12. Brasil. Lei n 8.842, de 4 de janeiro de 1994. Dispõe sobre a Política Nacional do Idoso e dá outras providencias. Diário Oficial da República Federativa do Brasil. Disponível em: http://www.pge.sp.gov.br/centrodeestudos/ bibliotecavirtual/dh/volume\%2Oi/idosolei8842. htm

13. Camarano AA. Envelhecimento da população brasileira: uma contribuição demográfica. In: Freitas EV et al. Tratado de geriatria e gerontologia. Rio de Janeiro: Guanabara Koogan; 2006. p. 80-105.

14. Merhy EE. A perda da dimensão cuidadora na produção da saúde: uma discussão do modelo assistencial e da intervenção no seu modo de trabalhar a assistência. In: Campos CR et al., organizadores. Sistema Único de Saúde em Belo Horizonte: reescrevendo o público. São Paulo: Xamã; 1998. p. 103-120. Disponível em: http:// www.hc.ufmg.br/gids/anexos/perda.pdf.

15. Brasil. Ministério da Saúde. Portaria $n^{\circ} 2.528$, de 19 de outubro de 2006. Aprova a Política nacional de Saúde da Pessoa Idosa. Diário Oficial da União, Brasília, DF, 20 de out. 2006. Disponível em: http://www.iea.usp.br/ politicanacionaldesaudedapessoaidosa.pdf

16. Bahia. Secretária da Saúde do Estado da Bahia. Guia do trabalhador para acolhimento pedagógico. Salvador; 2009.

17. Chaimowicz F. Epidemiologia e o envelhecimento no Brasil. In: FREITAS EV et al. Tratado de geriatria e gerontologia. Rio de Janeiro: Guanabara Koogan; 2006. p. 1152-1159.

18. Merhy EE, Franco TB, Bueno WS. O acolhimento e os processos de trabalho em saúde: o caso de Betim, Minas Gerais, Brasil. Cad. saúde pública. 1999;15(2):345-353.

19. Brasil. Ministério da saúde, Secretária de Atenção a Saúde, Departamento de Atenção Básica. Portaria n 648/GM, de 28 de março de 2006. Aprova a Política nacional de Atenção Básica para o Programa de Saúde da Família (PSF) e o Programa Agentes Comunitários de Saúde (PACS). Brasília, DF; 2006. Disponível em: http://www.saude.sc.gov.br/gestores/Pacto_ de_Gestao/portarias/GM-648.html

20. São Paulo. Secretária Municipal da Saúde. Coordenadoria de desenvolvimento e políticas 
de saúde. Caderno de violência contra a pessoa idosa: orientações gerais. São Paulo; 2007.

21. Brasil. Ministério da Saúde. Secretaria de Atenção à Saúde. Núcleo Técnico da Política Nacional de Humanização. Acolhimento nas práticas de produção de saúde. Brasília, DF; 2006. Disponível em: http://portal.saude.gov.br/ portal/arquivos/pdf/APPS_PNH.pdf
22. Teixeira RR. $\mathrm{O}$ acolhimento num serviço de saúde entendido como uma rede de conversações. In: Pinheiro R; Mattos RA. Construção da integralidade: cotidiano, saberes e práticas em saúde. Rio de Janeiro: Instituto de Medicina Social; Universidade do Estado do Rio de Janeiro; ABRASCO; 2003.p. 4961. Disponível em: http://www.corposem.org/ rizoma/acolhiconversa.htm 